\title{
A Comparison of Two Dynamic Mesh Methods in Fluid -Structure Interaction
}

\author{
JIA Huan ${ }^{a}$, SUN Qin ${ }^{b}$ \\ School of Aeronautics, Northwestern Polytechnical University, Xian china \\ aKillerking.love@163.com, bsunqin@nwpu.edu.cn
}

Keywords: fluid-structure interaction; Radial basis function; Delaunay; dynamic mesh.

\begin{abstract}
Two dynamic mesh methods - Radial Basis Function (RBF) method and Delaunay method which have been used widely in fluid-structure interaction recently were compared. Primarily, the basic theory of these two methods and the dynamic mesh process were presented. Furthermore, using the variable bandwidth sparse matrix storage technique, the computational efficiency of the RBF method can be improved. Taking ONERA M6 wing as an example, the mesh quality and computation time of the mesh motion after the airfoil rotation and wing deformation were checked. The results show that RBF method generate a better quality mesh in both structure and unstructured mesh while Delaunay method performed high efficiency, especially when the node number of the wing surface is large.
\end{abstract}

\section{Introduction}

In fluid-structure interaction, mesh motion is not only relate to the consumption of computer time but also affect the calculation accuracy, especially in the CSD/CFD weak coupling method of static aeroelasticity. In this process, the spatial grid of the fluid field must adjust according to the wing deformation which is transferred from the finite element model. If the spatial grid remeshed after the wing deformation each time, much computation time will be wasted and the load-displacement transfer will also be interrupted. From this point, the dynamic mesh[1] which keeps the node number and index unchanged is preferred. The common dynamic mesh methods used in commercial software such as FLUNT, CFX are spring[2] and elastic volume method[3]. Although these two methods have applied in many field, the computer time and storage consumed greatly. Moreover, the low quality grid or even negative volume grid will accrue when the deformation is large and harsh.

Recently, the Radial basis function method (RBF)and the Delaunay method have been used in the fluid-structure interaction filed. Relatively tradional method, these two methods can compute the mesh motion quickly while the mesh quality kept. RBF[4] method, a pure interpolation method based on scattered points, was first proposed by C.B.Allen[5] in 2008. The advantages such as mesh type independence and smooth deformation shape made it popular in mesh motion. Delaunay method is a common rule in meshing which applied in computer graphics mostly. In 2006 LIU[6] introduced Delaunay method in mesh motion. By adding some auxiliary boundary points, XIAO Tian Hang[7] improved this method further. Although these two methods have been applied in mesh motion widely, no paper describe the comparison of these two methods.

In this paper, we consider two tests in ONERA M6 static aeroelasticity which need the mesh motion properly. Both structure and unstructured mesh will be used in these tests. At last, we will get the advantages of each method and their applied range.

\section{Radial Basis Function}

The form of RBF method used in mesh motion is:

$$
S(\mathrm{r})=\sum_{i=1}^{N} \alpha_{i} \phi\left(\left\|\mathrm{r}-\mathrm{r}_{i}\right\|\right)
$$


Where $s(r)$ is the interpolation function of spatial position $r$. In this case, it stands for node displacement. $\phi$ is basis function adopted and $\| \bullet \mid$ take 2-norm.

The coefficients $\alpha_{i}$ can compute form interpolation condition:

$$
s\left(\mathrm{r}_{j}\right)=g_{j} \quad(\mathrm{j}=1, \cdots, \mathrm{N})
$$

Where $g_{i}$ is the function value stand for the wing surface node displacement and $\mathrm{N}$ is the node number.

Using $s$ to denote a surface node. For given surface node coordination $r_{s j}$ and displacement $\Delta x_{s j}$, taken $x$ direction as an example, the equation (1) can write in matrix form:

$\Delta \mathrm{x}_{S}=\mathrm{M} \mathrm{a}_{x}$

Where,

$$
\begin{aligned}
& \Delta \mathbf{x}_{s}=\left(\begin{array}{c}
\Delta x_{s 1} \\
\vdots \\
\Delta x_{s N}
\end{array}\right), \mathbf{a}_{x}=\left(\begin{array}{c}
\alpha_{s 1}^{x} \\
\vdots \\
\alpha_{s N}^{x}
\end{array}\right) \quad M=\left(\begin{array}{cccc}
\phi_{s 1 s 1} & \phi_{s 1 s 2} & \cdots & \phi_{s 1 s N} \\
\vdots & \vdots & \ddots & \vdots \\
\phi_{s N s 1} & \phi_{s N s 2} & \cdots & \phi_{s N S N}
\end{array}\right) \text { and } \\
& \phi_{s i s j}=\phi\left(\left\|r_{s i}-\mathrm{r}_{s j}\right\| / R\right)
\end{aligned}
$$

The compact radius $R$, usually takes five to ten times of the wing maximum deformation. By computing the inverse matrix $\mathrm{M}$, the interpolation coefficient $\alpha_{i}$ can get from equation (3).

By substituting wing surface node location into equation (1), the spatial node location after wing deformation will be determined as equation (4):

$$
\Delta x_{V}=\mathrm{A} \mathrm{a}_{x}=\mathrm{AM}^{-1} \Delta x_{s}=\mathrm{H} \Delta x_{s}
$$

Where,

$$
A=\left(\begin{array}{cccc}
\phi_{v 1 s 1} & \phi_{v 1 s 2} & \cdots & \phi_{v 1 s n} \\
\vdots & \vdots & \ddots & \vdots \\
\phi_{v n s 1} & \phi_{v n s 2} & \cdots & \phi_{v n S N}
\end{array}\right), v \text { stand for spatial node and } n \text { is spatial node number. }
$$

The most time consuming process of RBF method is computing inverse matrix $\mathrm{M}$. Since $\mathrm{M}$ is a sparse matrix, a method based on variable bandwidth storage will be introduced to reducing the computation time. Using a one-dimensional array A to record the first nonzero element index of each line of $\mathrm{M}$ and a one-dimension array $\mathrm{B}$ to record the elements form the first nonzero to diagonal element of each line. Then, using symmetric decomposition method to solve linear equations instead of solving the inverse matrix $\mathrm{M}$ directly. This method will reduce almost $80 \%$ computer storage and improve the compute efficiency.

\section{Delaunay Method}

Delaunay method used in mesh motion divide into four steps:

First, we generate the Delaunay graph according to the geometry boundary. The Delaunay criterion is that the circum-circle for triangles or the circum-for tetrahedron should not include other points except the points which construct the triangular or tetrahedron. If the geometry boundary is convex, this mesh can always proceed and unique. Usually, wing surface and far boundary points take as geometry to generate the Delaunay triangular.

After the Delaunay triangular generation, all spatial nodes should locate in its Delaunay triangular. For a point p, search the triangular which contain this point and calculate the surface or volume coordinates as equation (5), (6).

$$
\begin{aligned}
& e_{i}=\frac{S_{i}}{S} \quad i=1,2,3 \\
& e_{i}=\frac{V_{i}}{V}
\end{aligned}
$$


The key step in Delaunay method is moving the Delaunay triangular base on boundary deformation. All the connectivity and vertex index should be kept. If the deformation is too large, triangular deformation may failure. In this case, split the deformation into two shamll steps and go back to step 1 regenerating the Delaunay triangular.

At last, relocate the spatial node. According to the surface or volume coordinate at step2, turn them into Cartesian at the moved triangular. As equation (7) show:

$$
x_{p}^{\prime}=\sum_{i=1}^{4} e_{i} x_{i}^{\prime}
$$

\section{Test Cases}

\section{Airfoil Rotation}

In this case, naca0012 airfoil will rotate about its back edge 30 degrees. All the fluid uses a structure mesh with lowest quality 0.7 . The surface girds are quad with 200 nodes and spatial grids are hexahedron with 40325 nodes. Figure 1 demonstrates the mesh before deformation.

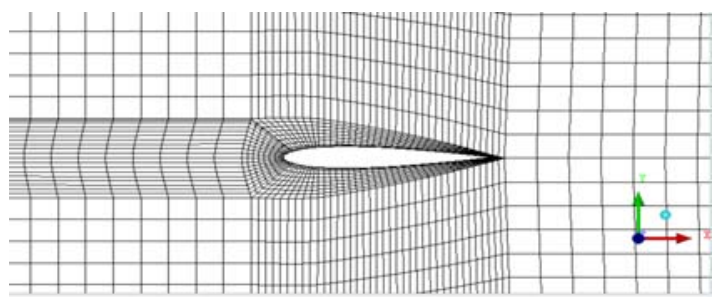

Figure 1 mesh before deformation

For Delaunay method, first step is generating Delaunay triangular according to geometry boundary as figure2 show:

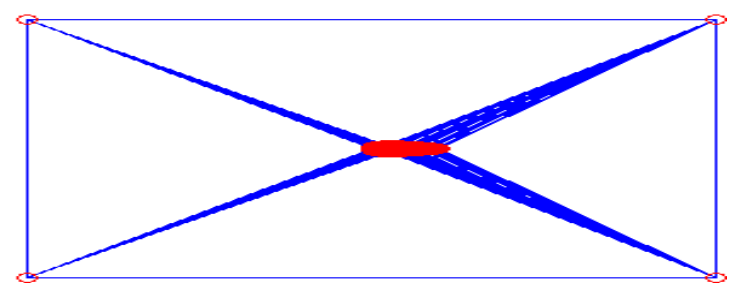

Figure 2 initial Delaunay triangular

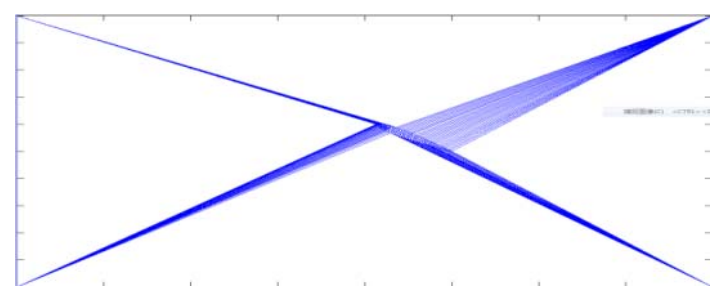

Figure 3 Delaunay triangular after deformation

Then, compute the surface coordinates of spatial nodes in Delaunay triangular.

After the geometry deformation, the Delaunay triangular will deform as figure 3.

By keeping the surface coordinates unchanged, we relocate the spatial nodes in Cartesian coordinates. Figure 4 show the mesh after deformation.

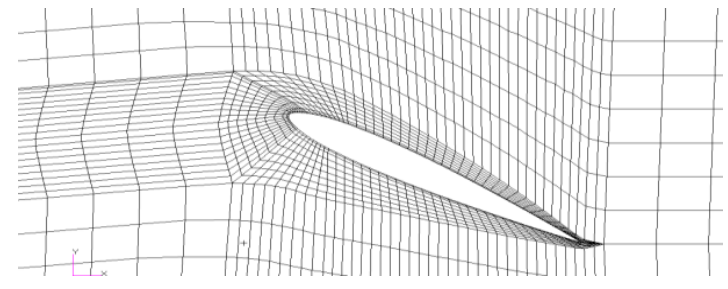

Figure 4 dynamic mesh by Delaunay

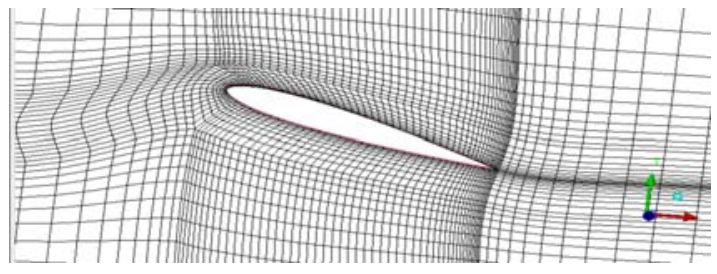

Figure 5 dynamic mesh by RBF

For RBF method, once we get interpolate coefficient $\alpha_{i}$ based on current airfoil rotation , the spatial node coordinates will be determined too. Figure 5 show the RBF dynamic mesh after deformation.

There are 13 elements whose quality below 0.7 in RBF and 116 elements in Delaunay method. RBF method use $5 \mathrm{~s}$ in mesh motion while Delaunay takes 4.2s.

The result show that both methods keep well mesh quality after airfoil rotation. RBF generate a better mesh due to the smooth function $s(x)$. Though Delaunay method shows slight in efficiency, 
Delaunay method may failure because of skewness triangular after the deformation if the airfoil rotates too much.

\section{Wing Deformation}

Take ONERA M6 wing unstructure mesh as an example, the computational gird that has 4373 nodes on wing surface triangular mesh and 116911 nodes in spatial tetrahedron mesh. 2965 elements mesh quality below 0.3 . Surface and spatial mesh demonstrate in figure 6 , 7 . Wing deformation is shown in figure 8.

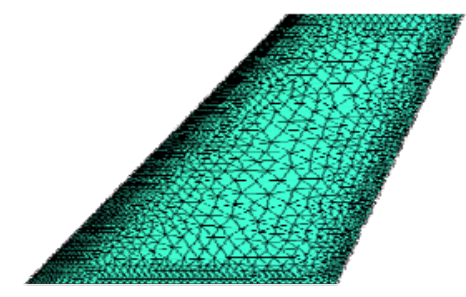

Figure 6 surface mesh

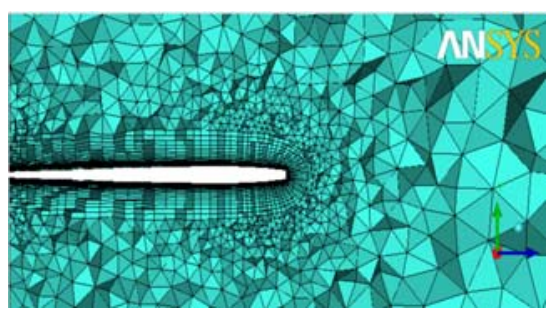

Figure 7 spatial mesh

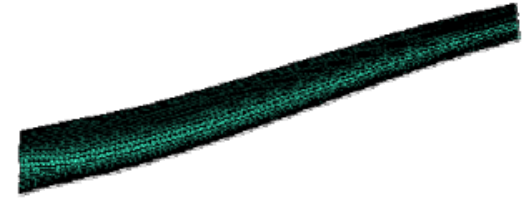

Figure 8 wing deformation

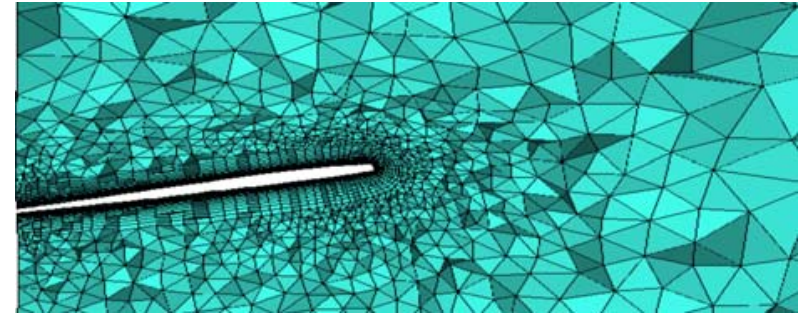

Figure 9 dynamic mesh by Delaunay

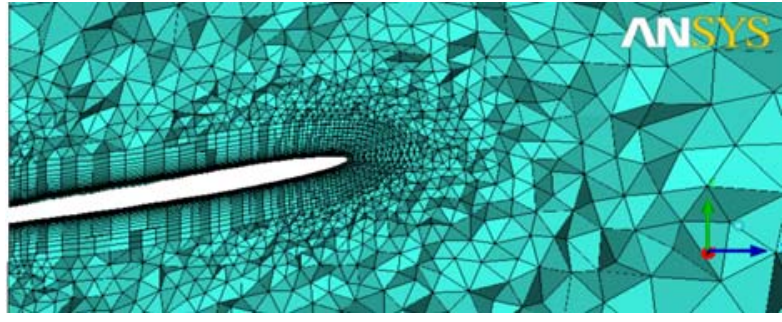

Figure 10 dynamic mesh by RBF

The mesh after deformation are shown as figure 9, 10. During wing mesh deformation, RBF take 72.8s while 3703 grids mesh quality below 0.3. Delaunay takes 9.7s while 5969 grids mesh quality below 0.3 .

In this case, RBF method still get a better quality mesh but consume almost ten times computer time than Delaunay method. Too much time spend on solving inverse matrix $\mathrm{M}$. The more the surface node is, the more time it takes. Table 1 show the computer time these two method consumed at difference wing surface numbers

Table 1 computer time of RBF and Delaunay method

\begin{tabular}{|l|l|l|l|l|l|l|}
\hline Wing node number & 200 & 1000 & 2000 & 4000 & 8000 & 10000 \\
\hline RBF [s] & 5 & 13 & 27 & 72 & 500 & 900 \\
\hline Delaunay [s] & 4 & 5 & 7 & 9 & 14 & 30 \\
\hline
\end{tabular}

\section{Conclusion}

In this paper, a comparison of RBF and Delaunay method show that when the surface node number is small RBF method is prefer due to the high quality mesh. On the contrary, the Delaunay is more efficient if the surface node number is large. In fact, RBF method may take too much time than generate a new mesh in this case.

\section{Reference}

[1] SHI Zhong jun,Xu Min,CHEN Shi lu. Analysis of Moving Mesh Generation Technology [J] JOURNAL OF AIR FORCE ENGNEERING UNIVERSITY (NATURAL SCIENCE EDITION) 4(1), February 2003,p:61-64

[2] J.T. Batina. Unsteady Euler algorithm with unstructured dynamic mesh for complex-aircraft aerodynamic analysis.[J] AIAA Journal, 29(3),April 1991,p:327-333 
[3] Bar-Yoseph P Z, Mersu S, Chippada S,et al. Automatic monitoring of element shape quality in 2D and 3D computational mesh dynamics[J]. Computational Mechanics, 2001, 27(5):378-395

[4] ZHU Wen wen. Scattered Data Interpolation with Random Radical Basis Functions [D].Liaoning Normal University, May, 2009,p:8-12

[5] C.B Allen, T.C.S. Rendall Unified Approach to CFD-CSD Interpolation and Mesh Motion using Radial Basis Functions[A] 25th AIAA Applied Aerodynamics Conference. p,25-28 June 2007, Miami, FL

[6] Liu X Q, Qin N Xia H. Fast dynamic grid deformation based on Delaunay graph mapping [J]. Journal of Computational Physics, 11(2) February 2006,p:405-423

[7] Xiao Tianhang, Ang Haisong,Tong Chao. A New Dynamic Mesh Generation Method for Large Movements of Flapping wings with Complex Geometries [J].ACTA AEROAUTICA ET ASTRONAUTICA SINICA, 29(1) January 2008.p:41-48. 